\title{
Participation of autophagy in the cytotoxicity against breast cancer cells by cisplatin
}

\author{
MENG SHEN $^{1 *}$, WEI-MING DUAN $^{1 *}$, MENG-YAO WU ${ }^{1}$, WEN-JIE WANG ${ }^{1}$, LU LIU $^{1}$, MENG-DAN XU $^{1}$, JIE ZHU ${ }^{1}$, \\ DAO-MING LI ${ }^{1}$, QI GUI ${ }^{1}$, LIAN LIAN ${ }^{1,2}$, FEI-RAN GONG ${ }^{3}, \mathrm{KAI} \mathrm{CHEN}^{1}$, WEI LI ${ }^{1}$ and MIN TAO ${ }^{1,4}$ \\ ${ }^{1}$ Department of Oncology, The First Affiliated Hospital of Soochow University, Suzhou, Jiangsu 215006; \\ ${ }^{2}$ Department of Oncology, Suzhou Xiangcheng People's Hospital, Suzhou, Jiangsu 215131; \\ ${ }^{3}$ Department of Hematology, The First Affiliated Hospital of Soochow University, Suzhou, Jiangsu 215006; \\ ${ }^{4}$ Jiangsu Institute of Clinical Immunology, Suzhou, Jiangsu 215006, P.R. China
}

Received February 11, 2015; Accepted April 30, 2015

DOI: 10.3892/or.2015.4005

\begin{abstract}
Breast cancer is one of the most common cancers affecting women worldwide. Conventional chemotherapy is still one of the major approaches to the treatment of breast cancer. Autophagy, also termed as type II programmed cell death (PCD), exhibits either a protumorigenic or antitumorigenic function. In the present study, we investigated whether autophagy could be involved in the effect of chemotherapy against breast cancer. Epirubicin, docetaxel, methotrexate, cyclophosphamide, fluorouracil (5-FU) and cisplatin were applied in the present investigation. All of these chemotherapeutics presented cytotoxicity against breast cancer cells. DsRed-LC3 reporter assay revealed that only docetaxel and cisplatin induced autophagy. Autophagy inhibitor 3-methyladenine (3-MA) strengthened the cytotoxicity of docetaxel, yet impaired the cytotoxicity of cisplatin, suggesting that docetaxel stimulates protumorigenic autophagy, while cisplatin-induced autophagy could be antitumorigenic. Real-time PCR revealed that cisplatin upregulated multiple autophagy-related genes, including AMBRA1, ATG3, ATG4C, ATG4D, ATG5, ATG7, ATG13, ATG14, ATG16L2, Beclin1, DRAM1, GABARAP, GABARAPL1, GABARAPL2, HDAC6, IRGM, MAP1LC3B
\end{abstract}

Correspondence to: Dr Wei Li or Dr Kai Chen, Department of Oncology, The First Affiliated Hospital of Soochow University, Suzhou, Jiangsu 215006, P.R. China

E-mail: liwei10@suda.edu.cn

E-mail:kaichen@suda.edu.cn

*Contributed equally

Abbreviations: PCD, programmed cell death; DOC, docetaxel; MTX, methotrexate; CTX, cyclophosphamide; 5-FU, fluorouracil; DDP, cisplatin; ER, estrogen receptor; ATG protein, autophagyrelated protein; 3-MA, 3-methyladenine; MTT, methyl thiazolyl tetrazolium; DMSO, dimethyl sulfoxide; PI, propidium iodide; SD, standard deviation; PI3P, phosphatidylinositol-3-phosphate; PE, phosphatidylethanolamine

Key words: autophagy, chemotherapy, cisplatin, breast cancer and ULK1, indicating that cisplatin induced autophagy through a multiple mechanism involved manner.

\section{Introduction}

Breast cancer is one of the most common cancers affecting women worldwide, accounting for almost $23 \%$ of all cancers diagnosed in women (1-2). Although early detection, precise resection using wide margins and systematic adjuvant therapy have improved survival, breast cancer remains one of the leading causes of death among women (3). Conventional chemotherapy is still one of the major approaches to the treatment of breast cancer, particularly for cases of breast cancer in stages 2-4, and is particularly beneficial in estrogen receptor-negative $\left(\mathrm{ER}^{-}\right)$disease (4). Most chemotherapy medications work by destroying fast-growing and/or fast-replicating cancer cells, either by causing DNA damage upon replication or by other mechanisms.

Autophagy, also termed as type II programmed cell death (PCD), is a genetically programmed, evolutionarily conserved process that degrades long-living cellular proteins and organelles, including the endoplasmic reticulum, Golgi apparatus and mitochondria (5). Autophagy is important in the normal development and cellular response to environmental stimuli. In addition, autophagy participates in numerous diseases, including bacterial and viral infections, neurodegenerative disorders, cardiovascular diseases and cancers (5). Autophagy exhibits either a protumorigenic or antitumorigenic function, depending on the cell type, developmental stage of cancer and stimulator. Mechanistically, autophagy begins with sequestering cytoplasmic proteins or organelles in a membrane vacuole to form an autophagosome. Autophagosome formation is mediated by a set of evolutionarily conserved autophagy-related proteins (ATG proteins) (6). Previously, we demonstrated that stimulation of autophagy could be a potential strategy for the treatment of breast cancer (5).

In the present study, we investigated whether autophagy could be involved in chemotherapy against breast cancer and whether this participation could be protumorigenic or antitumorigenic. Moreover, mechanisms involved in this autophagic cell death by chemotherapeutics were also investigated. 
Table I. Primers.

\begin{tabular}{lllc}
\hline Genes & \multicolumn{1}{c}{ Sense (5'-3') } & \multicolumn{1}{c}{ Antisense (3'-5') } & $\begin{array}{c}\text { Product size } \\
(\text { bp) }\end{array}$ \\
\hline AMBRA1 & TCGGCAACAACATCATCGTC & CTGGGAAGGGAAACAGGAAT & 253 \\
ATG3 & ACTGATGCTGGCGGTGAAG & GTGGCAGATGAGGGTGATTT & 208 \\
ATG4A & GGGATGTATGCTACGCTGTGG & CACCCATTGTGCCATTTGAT & 182 \\
ATG4B & TAGGCCGAGATTGGAGGTG & GCGCTATCTGGTGAATGGAGT & 114 \\
ATG4C & TTTCCCTCTTGAGACATTCCAC & GGTGATTTCTTCAGAAGCTCGTT & 130 \\
ATG4D & AGCCGAGTGGAAGTCTGTGGT & AGCAGGAAGTCATCTTGGTAGCC & 177 \\
ATG5 & ATCAGGTTTGTGGAGGCA & GGTTAATGATGGCAGTGGAGG & 140 \\
ATG7 & CCAAGGTCAAAGGACGAAGAT & GTACGGTCACGGAAGCAAAC & 156 \\
ATG9A & CCTTACCTCACCCGCAGTTC & GGCAGCAAAGTATTTCCATCC & 241 \\
ATG9B & CAGCCGAACACCAAAGTCAT & GCTTCCCTTCCCTCTGTAAATC & 239 \\
ATG10 & ACACTATTACGCAACAGGAACATC & CTCAGAGGTAGATTCAGCCCAAC & 184 \\
ATG12 & ACCCATTGCTCCTACTTGTTACTAT & TTTCTGCCTGGTGGACTGC & 256 \\
ATG13 & TGTCATTGCTGCTGAAGTCCC & CCCACTGTCCCAACACGAACT & 169 \\
ATG14 & GTGAGCCGAGATTGTGCCAT & GGTAATAATGCCTGTTAGGACTCTTTC & 267 \\
ATG16L1 & GGGATTTCTGAAGATTTGACTGAG & ACCGACTTTGGAAGGACGAG & 248 \\
ATG16L2 & ACCGGACAGTGAAGGAGTGG & GGATCTTCTGGTCATTGTGGC & 129 \\
Beclin1 & GCTGGATGATGAGCTGAAGAGT & GTGCCAGATGTGGAAGGTTG & 109 \\
DRAM1 & TCGTCAGCCGCCTTCATTAT & CGAAACATCCCACCAATCC & 262 \\
GABARAP & TCAAACACCACCTCCCTTATTC & TGCCAACTCCACCATTACCC & 94 \\
GABARAPL1 & GCCTGATCTGGACAAGAGGAAGT & ATGGTAGCACTGGTGGGAGG & 150 \\
GABARAPL2 & GTTGACATTGACAAACGGAAGTAC & CATAGTTAGGCTGGACTGTGGG & 150 \\
HDAC6 & CATCCGAACTCATACTCCTGTGC & TAAGACTGTGCTGGGCGTGAT & 136 \\
IRGM & CTTGCTGCTGCTCATTCTTTG & CGAGTCTGGAGTTGTTCGTTTC & 133 \\
LAMP1 & ACAACACGACGGTGACAAGG & TTCATCCCGAACTGGAAGAGC & 136 \\
MAP1LC3B & CGCATTTGCCATCACAGTTG & TAGGAGTCAGGGACCTTCAGC & 94 \\
RAB24 & CAGAAAGTGGCAGAGGATTACG & TGACTACCCAAGCCCAGAAAG & 199 \\
RGS19 & ATCTACACGCTCATGCACCG & GACAACAACACCTGAAGGGAAC & 175 \\
ULK1 & CAGCAAAGGCATCATCCACC & GAAGCCGAAGTCAGCGATC & 115 \\
WIPI1 & CTACCAACTACCTCCCTACCCAG & TGTCCACTGGATGACGCAAC & 154 \\
\hline & & & \\
\hline
\end{tabular}

\section{Materials and methods}

Cell line and cultures. The human breast cancer cell lines MDA-MB-231 and MDA-MB-453 were purchased from the American Type Culture Collection (ATCC; Manassas, VA, USA). Cells were maintained in RPMI-1640 medium, supplemented with $10 \%$ fetal calf serum (both from Gibco, Grand Island, NY, USA), $100 \mathrm{U} / \mathrm{ml}$ penicillin and $100 \mathrm{mg} / \mathrm{ml}$ streptomycin. The cultures were incubated at $37^{\circ} \mathrm{C}$ in a humidified atmosphere with $5 \% \mathrm{CO}_{2}$. Cells were passaged every 2-3 days to obtain exponential growth.

Reagents. 3-Methyladenine (3-MA) was purchased from Sigma (St. Louis, MO, USA). Epirubicin was purchased from Pfizer (Shanghai, China). Docetaxel and methotrexate (MTX) were purchased from Jiangsu Hengrui Medicine Co., Ltd. (Liangyungang, Jiangsu, China). Cyclophosphamide (CTX) was purchased from Baxter Oncology $\mathrm{GmbH}$ (Kantstrasse, Halle, Germany). Fluorouracil (5-FU) was purchased from Shanghai Xudong Haipu Pharmaceutical Co., Ltd. (Shanghai, China). Cisplatin was purchased from
Nanjing Pharmaceutical Factory Co., Ltd. (Nanjing, Jiangsu, China).

MTT assay. Cellular growth was evaluated by methyl thiazolyl tetrazolium (MTT) assay (7). Cells were seeded into 24-well tissue culture plates at $5 \times 10^{4}$ cells/well. After treatment, MTT (Sigma, St. Louis, MO, USA) was added to each well to a final concentration of $0.5 \mathrm{mg} / \mathrm{ml}$, followed by incubation at $37^{\circ} \mathrm{C}$ for $4 \mathrm{~h}$. The medium was then removed, and $800 \mu \mathrm{l}$ of dimethylsulfoxide (DMSO) was added/well. The absorbance in each well was measured at $490 \mathrm{~nm}$ using a microplate ELISA reader (Bio-Rad Laboratories, Hercules, CA, USA). The inhibition rate was calculated as follows: Inhibition rate $=[$ (mean control absorbance - mean experimental absorbance)/mean control absorbance] x $100(\%)$. The concentration that caused $50 \%$ growth inhibition $\left(\mathrm{IC}_{50}\right)$ was calculated by the modified Kärber's method (7) according to the formula: $\mathrm{IC}_{50}=\lg ^{-1}[\mathrm{Xk}-\mathrm{i}(\Sigma \mathrm{p}-0.5)]$, in which $\mathrm{Xk}$ represents the logarithm of the highest drug concentration; $i$, is that of the ratio of the adjacent concentration; and $\Sigma p$, is the sum of the percentage of growth inhibition at various concentrations. 
A
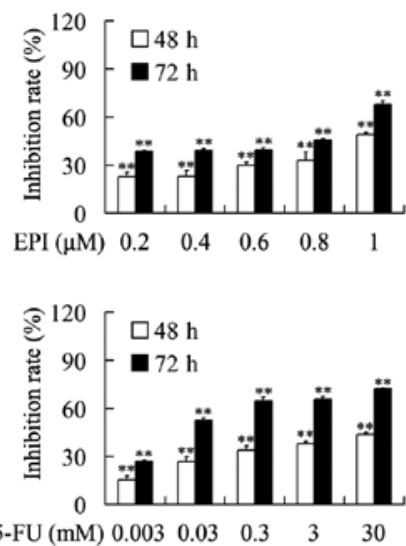

B
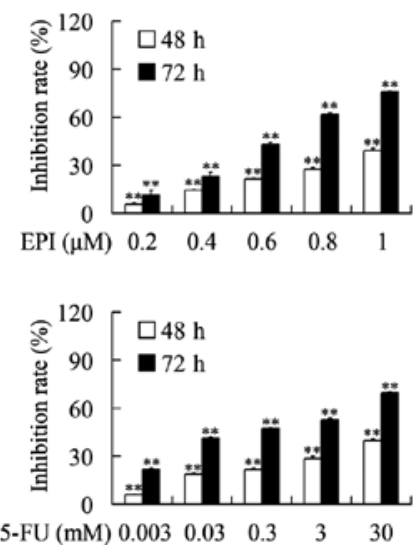
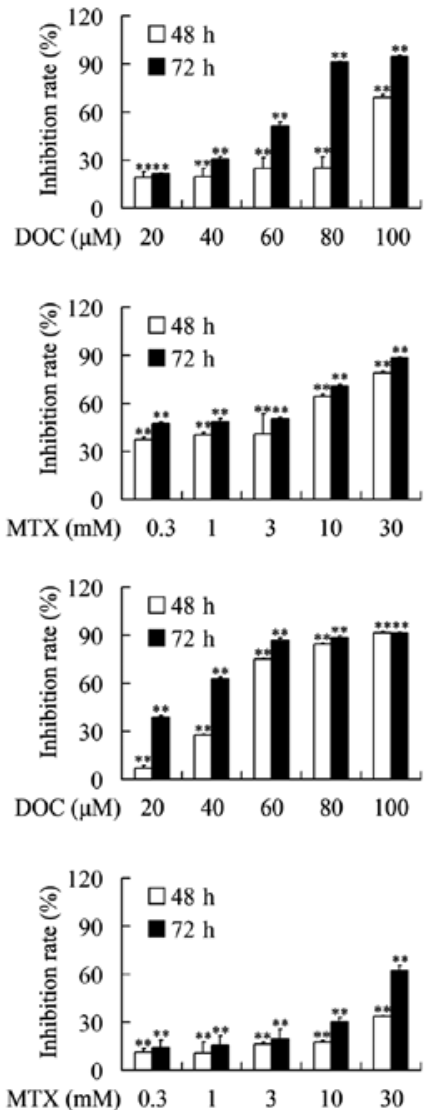
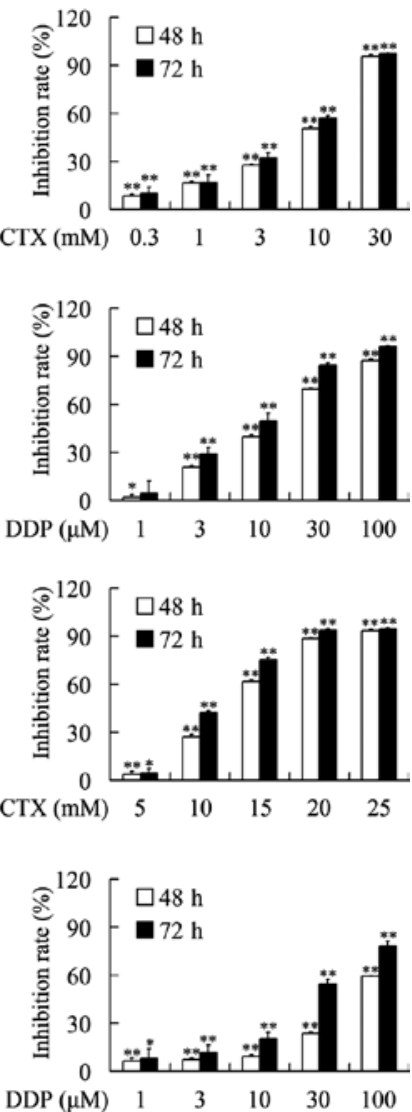

Figure 1. Cytotoxicity of epirubicin (EPI), docetaxel (DOC), methotrexate (MTX), cyclophosphamide (CTX), fluorouracil (5-FU) and cisplatin (DDP) on (A) MDA-MB-231 and (B) MDA-MB-453 breast cancer cells. ${ }^{*} \mathrm{P}<0.05,{ }^{* *} \mathrm{P}<0.01$ indicate significant difference from the respective control group.

Autophagy assay using the DsRed-LC3 reporter. Autophagy is dependent on the presence of autophagosomes and autolysosomes (8). LC3, the mammalian ontology of ATG8, is a credible marker for autophagosomes (9). During the formation of autophagosomes, LC3 will form punctate structures within the cytoplasm that correspond to autophagic vesicles $(8,9)$. To develop an autophagy reporter, we fused the LC3 cDNA to the $\mathrm{C}$ terminus of DsRed as previously described (10). To produce stable cell lines continuously reporting autophagy activity, recombinant lentiviruses expressing the DsRed-LC3 reporter were generated and used to infect the cells. The LC3 puncta were visualized with an Olympus BX51 microscope (Tokyo, Japan). DNA was counterstained using DAPI (Sigma).

Cell cycle analysis. Cell cycle analysis using propidium iodide (PI) was performed as previously described (7). Prior to treatment, cells were synchronized in the cell cycle by serum starvation for $24 \mathrm{~h}$. After treatment, the cells were fixed in $80 \%$ ice cold ethanol, and incubated with $0.5 \%$ Triton X-100 solution containing $1 \mathrm{mg} / \mathrm{ml}$ RNase A at $37^{\circ} \mathrm{C}$ for $30 \mathrm{~min}$. PI (Sigma) was added to a final concentration of $50 \mu \mathrm{g} / \mathrm{ml}$ followed by a 30 -min incubation in the dark. Cellular DNA content was analyzed by a fluorescence-activated cell sorter (FACS; Becton-Dickinson, Franklin Lakes, NJ, USA). Data were processed using ModFit LT software (Verity Software House, Inc., Topsham, ME, USA).

Real-time PCR. Total RNA was extracted using TRIzol reagent (Invitrogen, Carlsbad, CA, USA) according to the manufacturer's protocol. After spectrophotometric quantification, $1 \mu \mathrm{g}$ total RNA in a final volume of $20 \mu \mathrm{l}$ was used for reverse transcription with PrimeScript RT reagent kit (Takara, Otsu, Shiga, Japan) according to the manufacturer's protocol. Aliquots of cDNA corresponding to equal amounts of RNA were used for quantification of mRNA by real-time PCR using the LightCycler 96 real-time quantitative PCR detection system (Roche Diagnostics, Indianapolis, IN, USA). The reaction system $(25 \mu \mathrm{l})$ contained the corresponding cDNA, forward and reverse primers, and SYBR-Green PCR Master Mix (Roche Diagnostics). All data were analyzed using $\beta$-actin gene expression as an internal standard. The specific primers are presented in Table I.

Statistical analysis. Each experiment was performed a minimum of three times. Results are expressed as the mean value \pm standard deviation $(\mathrm{SD})$. Statistical analysis was performed using unpaired Student's t-test. The correlations between microarray assays were analyzed using Spearman's rank correlation analysis. A $\mathrm{P}$ value $<0.05$ was considered to indicate a statistically significant result.

\section{Results}

Cytotoxicity of chemotherapeutics against breast cancer cells. The growth inhibition effects of epirubicin, docetaxel, MTX, CTX, 5-FU and cisplatin against breast cancer cells were evaluated using MTT assays. As shown in Fig. 1, epirubicin, docetaxel, MTX, CTX, 5-FU and cisplatin suppressed 
A
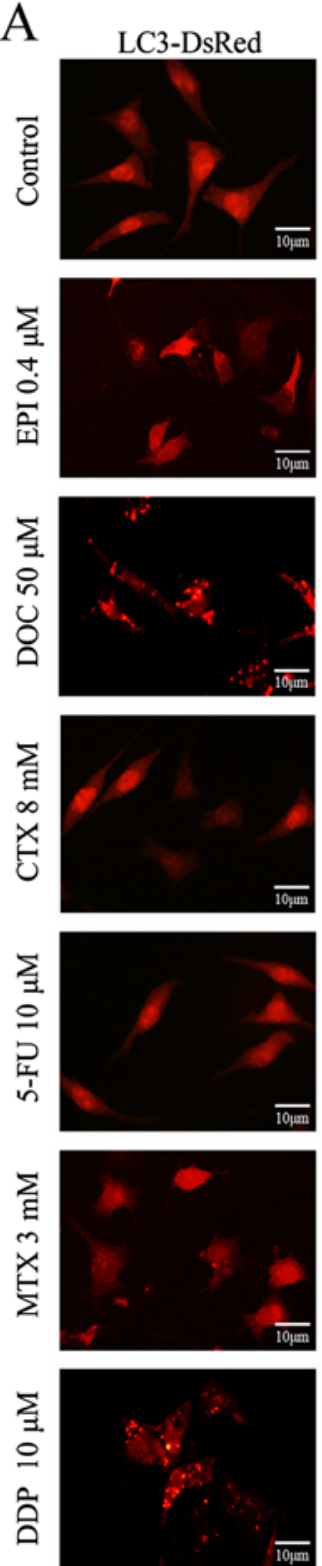

DAPI
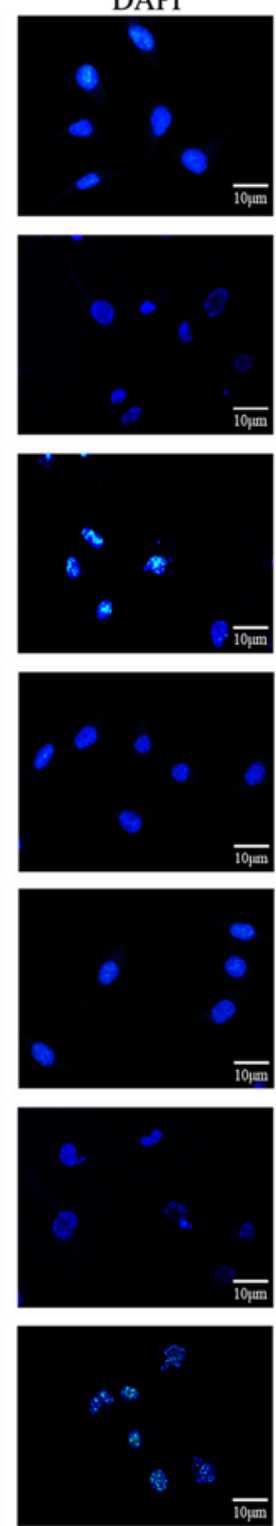
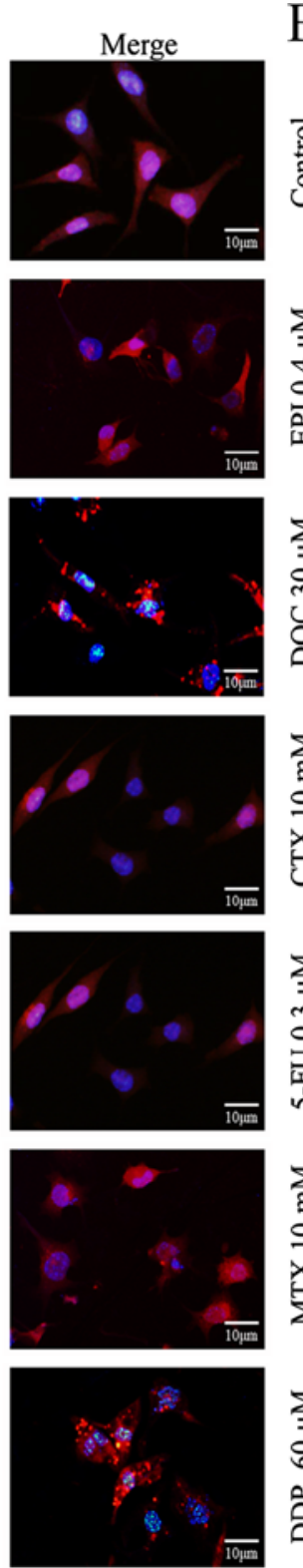

B
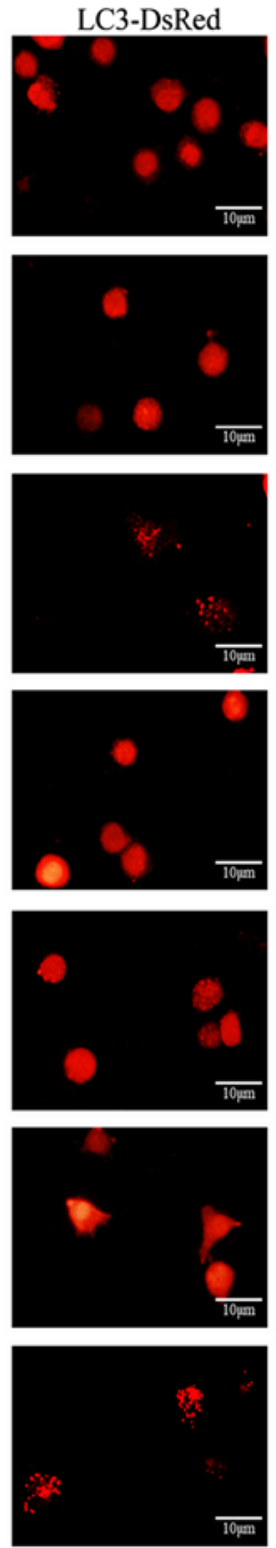

DAPI
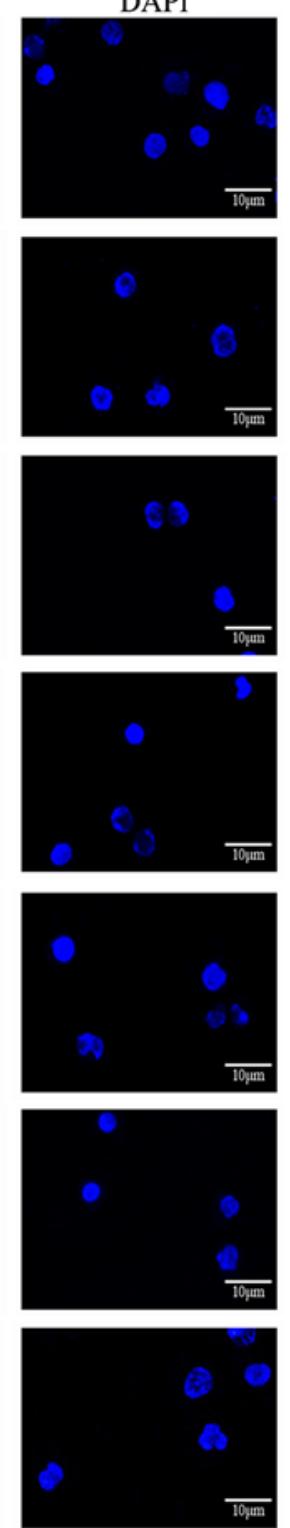

Merge
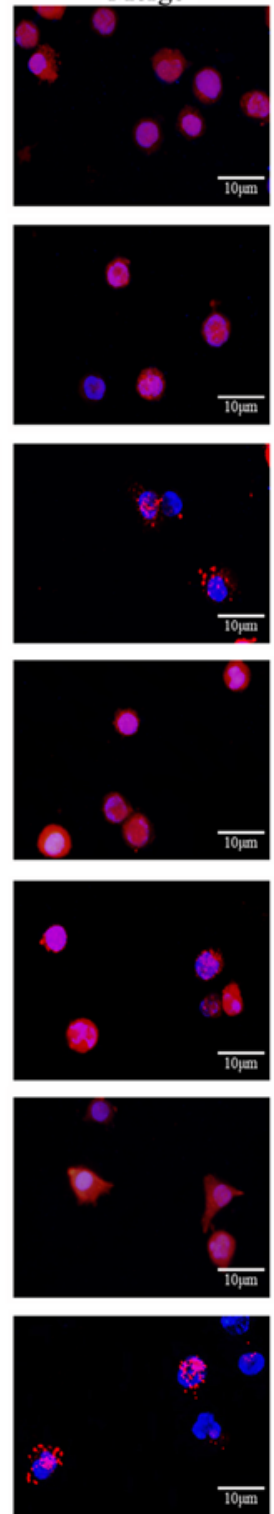

Figure 2. Formation of LC3 punctate after treatment with chemotherapeutics in (A) MDA-MB-231 and (B) MDA-MB-453 breast cancer cells. DsRed-LC3overexpressing breast cancer cells were treated with epirubicin (EPI), docetaxel (DOC), methotrexate (MTX), cyclophosphamide (CTX), fluorouracil (5-FU) or cisplatin (DDP) for $24 \mathrm{~h}$, followed by DsRed-LC3 reporting autophagy assay.

the growth of MDA-MB-231 and MDA-MB-453 cells in a dose- and time-dependent manner. The $\mathrm{IC}_{50}$ values after treatment with each chemotherapeutic for 48 and $72 \mathrm{~h}$ are presented in Table II.

Docetaxel and cisplatin induce autophagy in breast cancer cells. Using the DsRed-LC3 reporter, increased formation of LC3 punctate was observed following the treatment with docetaxel or cisplatin, yet not following other chemotherapeutics, suggesting docetaxel and cisplatin induced autophagy in the breast cancer cells (Fig. 2).

Cisplatin suppresses breast cancer growth through an autophagy-dependent manner. To verify whether the growth inhibition induced by chemotherapeutics was executed in an autophagy-dependent manner, we investigated the effect
Table II. $\mathrm{IC}_{50}$ values of the chemotherapeutics at 48 and $72 \mathrm{~h}$ in the MDA-MB-231 and MDA-MB-453 cells.

\begin{tabular}{lrrrrr}
\hline & \multicolumn{2}{c}{ MDA-MB-231 } & & \multicolumn{2}{c}{ MDA-MB-453 } \\
\cline { 2 - 3 } \cline { 5 - 6 } & \multicolumn{1}{c}{$48 \mathrm{~h}$} & $72 \mathrm{~h}$ & & $48 \mathrm{~h}$ & $72 \mathrm{~h}$ \\
\hline EPI & $1.28 \mu \mathrm{M}$ & $0.60 \mu \mathrm{M}$ & & $2.05 \mu \mathrm{M}$ & $0.61 \mu \mathrm{M}$ \\
DOC & $75.35 \mu \mathrm{M}$ & $45.45 \mu \mathrm{M}$ & & $46.39 \mu \mathrm{M}$ & $27.38 \mu \mathrm{M}$ \\
CTX & $5.02 \mathrm{mM}$ & $4.38 \mathrm{mM}$ & & $12.04 \mathrm{mM}$ & $10.70 \mathrm{mM}$ \\
5-FU & $119.33 \mathrm{mM}$ & $89.22 \mu \mathrm{M}$ & & $715.41 \mathrm{mM}$ & $603.47 \mu \mathrm{M}$ \\
MTX & $2.45 \mathrm{mM}$ & $1.10 \mathrm{mM}$ & & $724.39 \mathrm{mM}$ & $17.29 \mathrm{mM}$ \\
DDP & $13.48 \mu \mathrm{M}$ & $8.61 \mu \mathrm{M}$ & & $225.78 \mu \mathrm{M}$ & $72.94 \mu \mathrm{M}$ \\
\hline
\end{tabular}

EPI, epirubicin; DOC, docetaxel; CTX, cyclophosphamide; 5-FU, fluorouracil; MTX, methotrexate; DDP, cisplatin. 
A
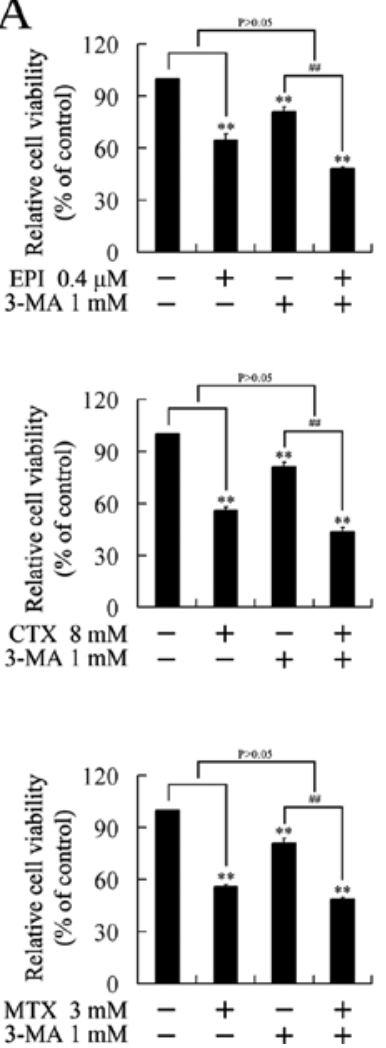
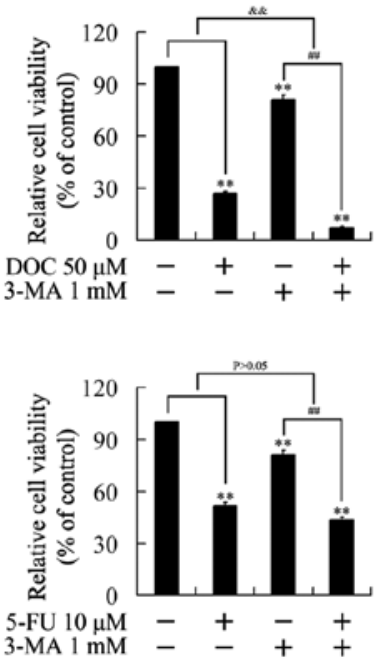

B
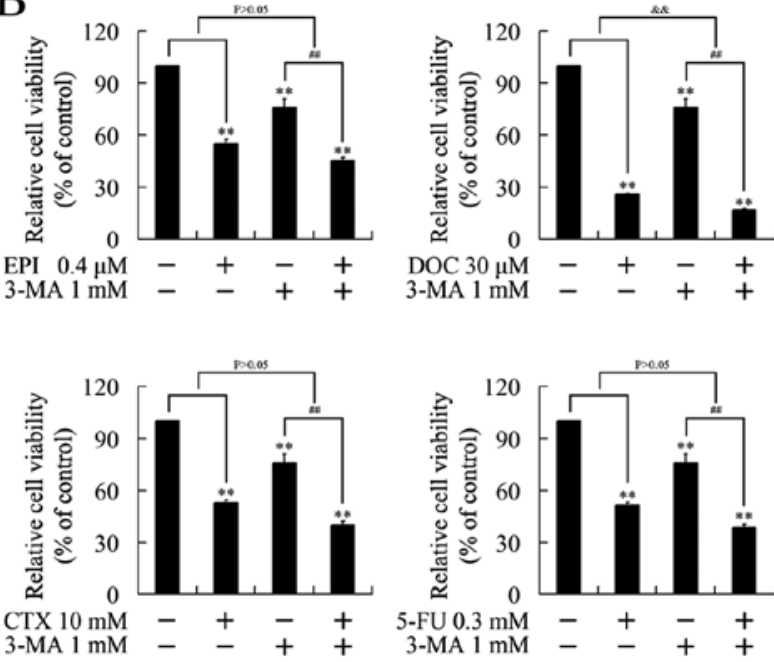

Figure 3. Involvement of autophagy in the cytotoxicity of chemotherapeutics against (A) MDA-MB-231 and (B) MDA-MB-453 breast cancer cells. ${ }^{* *} \mathrm{P}<0.01$ indicates significant differences from the respective control groups; ${ }^{\#} \mathrm{P}<0.05$ and ${ }^{\# \#} \mathrm{P}<0.01$ indicated significant differences from the respective 3 -MA groups; ${ }^{\&}$ \& $<0.01$ indicated significant differences between fold induction. EPI, epirubicin; 3-MA, 3-methyladenine; DOC, docetaxel; CTX, cyclophosphamide; 5-FU, fluorouracil; MTX, methotrexate; DDP, cisplatin.

A

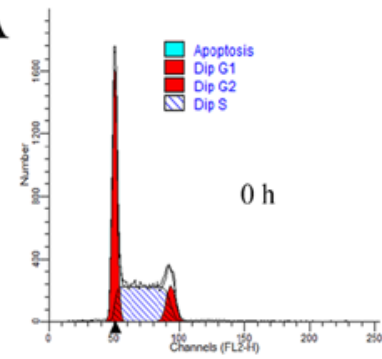

B

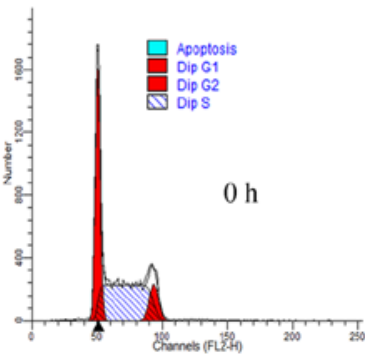

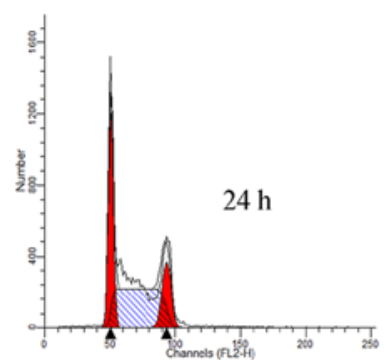
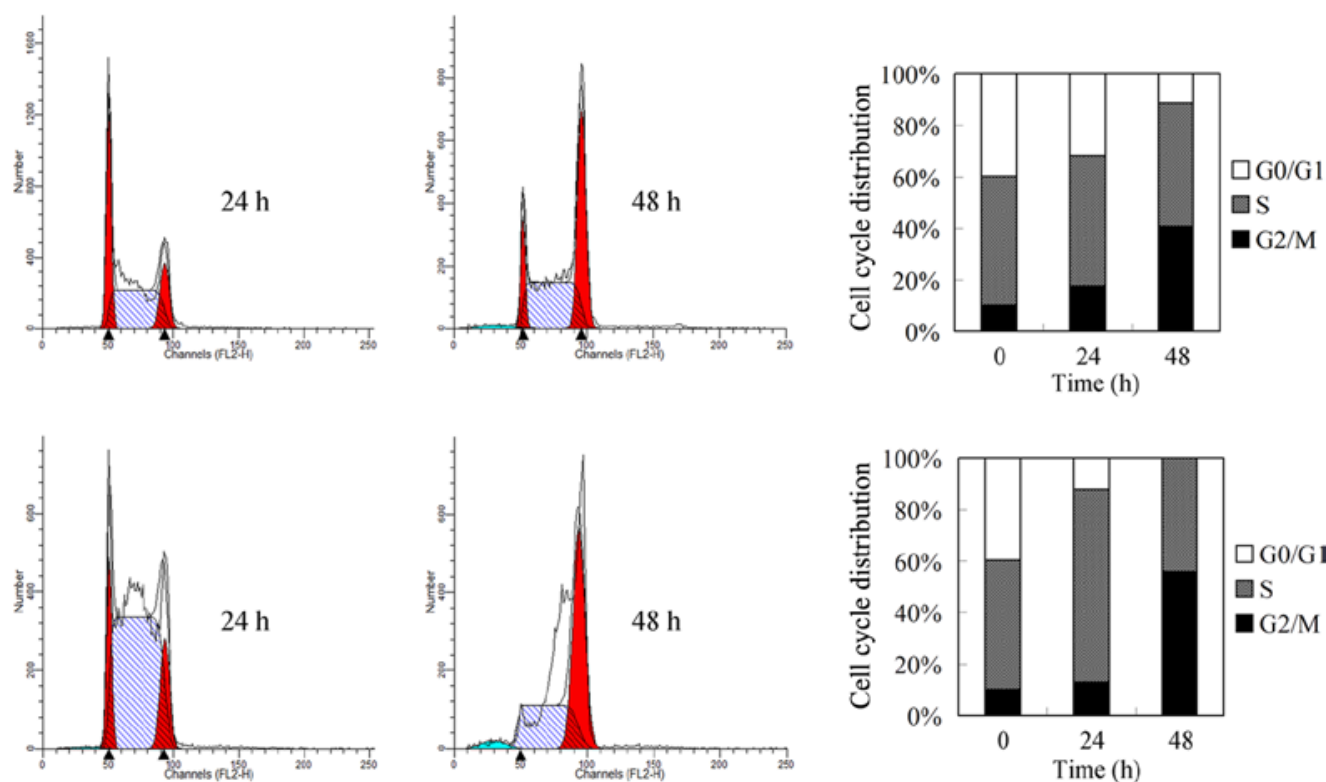
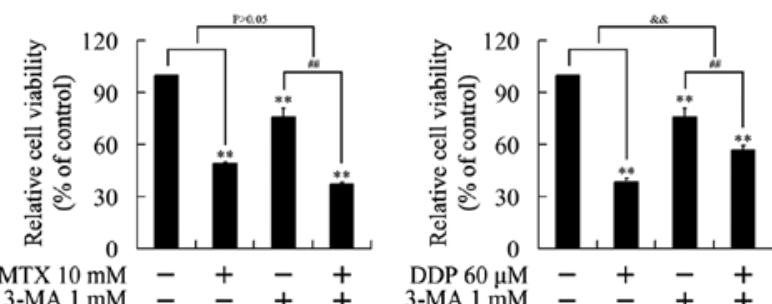

Figure 4. Cell cycle distribution after treatment with (A) docetaxel and (B) cisplatin.

of 3-MA, a classic autophagy inhibitor, on the cytotoxicity of the chemotherapeutics. As expected, 3-MA had no effect on epirubicin, MTX, CTX and 5-FU, consistent with the undetectable autophagy by these chemotherapeutics. 3-MA attenuated the cytotoxicity of cisplatin, suggesting that cisplatin suppressed cell growth in an autophagy-dependent manner. Notably, 3-MA strengthened the growth inhibition induced by docetaxel, indicating the autophagy induced by docetaxel impaired its cytotoxicity (Fig. 3).

Cell cycle regulation by docetaxel and cisplatin in breast cancer cells. Cell cycle arrest and autophagy are closely linked 
A
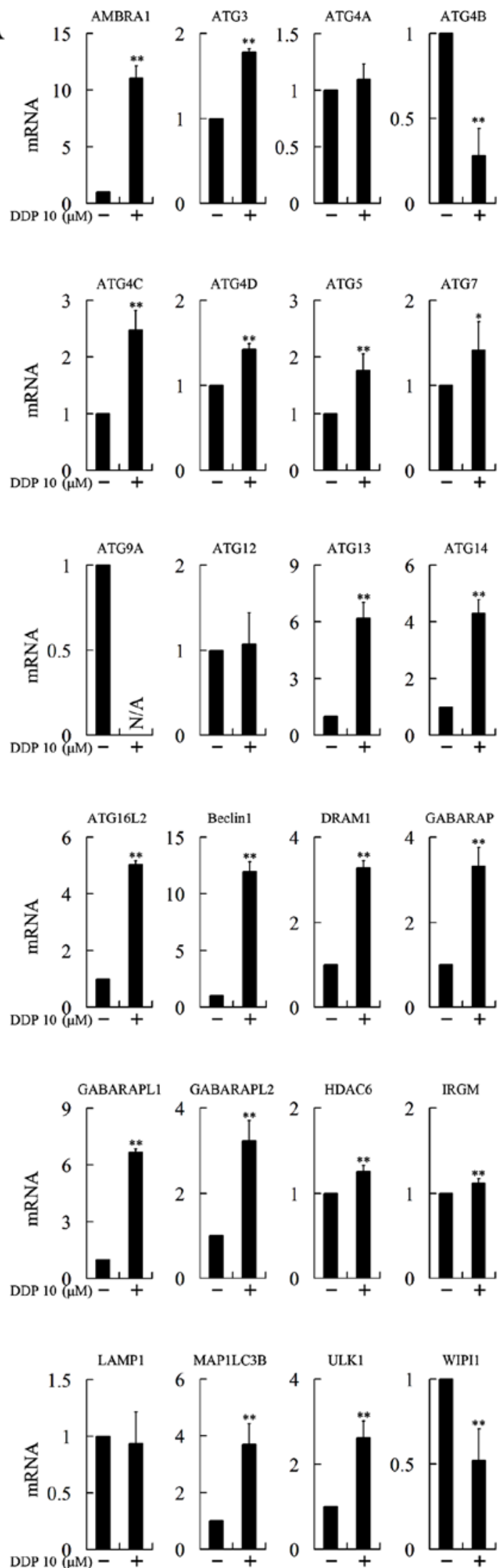

B
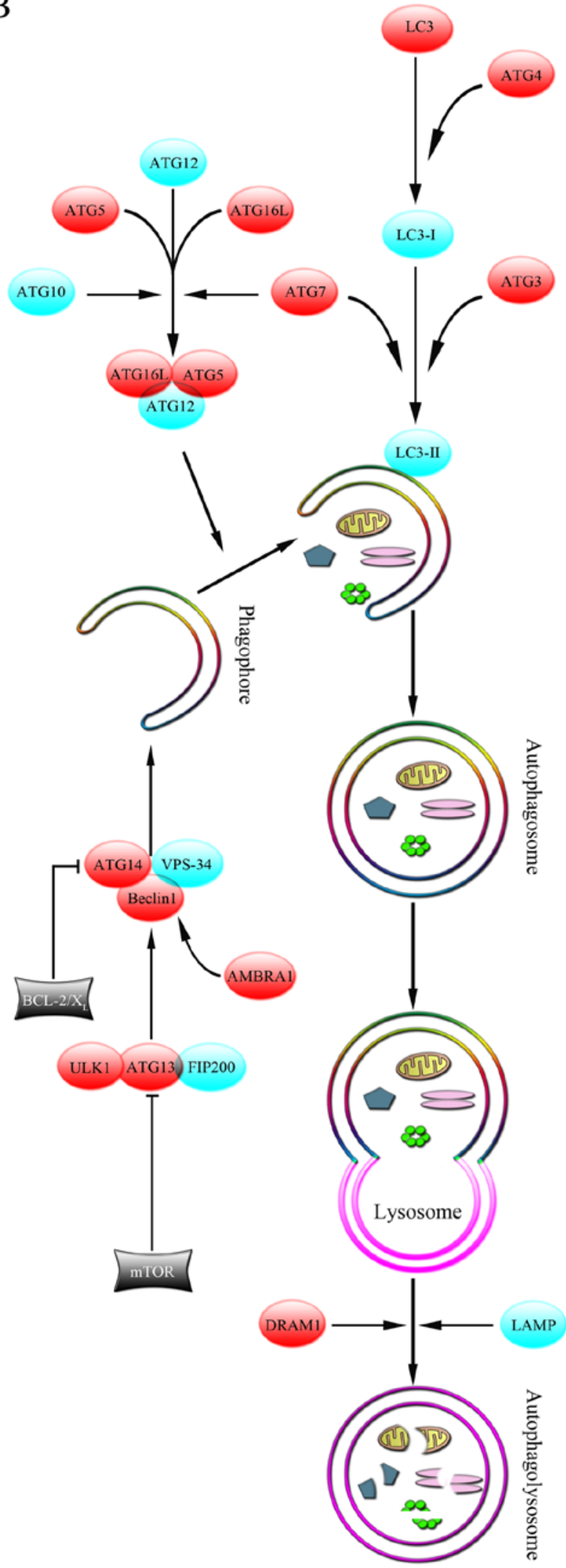

Figure 5. Potential mechanisms involved in cisplatin-induced autophagy. (A) Expression of autophagy-related genes after treatment with cisplatin. (B) Autophagic cell signaling transduction pathway. Upregulated genes following treatment with cisplatin are highlighted in red.

biological processes (11). We previously reported that autophagy induction results in $\mathrm{G} 2 / \mathrm{M}$ cell cycle arrest. To investigate the mechanism involved in docetaxel and cisplatin-induced growth repression, we carried out flow cytometric analysis. As shown in Fig. 4A, docetaxel induced G2/M cell arrest and decreased the population of cells in the G0/G1 phase. Cisplatin induced 
accumulation of cells in the S phase (Fig. 4B), consistent with its DNA crosslinking role (12). However, with prolonged treatment, an increased $\mathrm{G} 2 / \mathrm{M}$ population and annihilation of the $\mathrm{G} 0 / \mathrm{G} 1$ population were noted, suggesting that mechanisms beyond DNA crosslinking could be involved. Considering the $\mathrm{G} 2 / \mathrm{M}$ population accumulating role of autophagy, we speculated that autophagic cell death could be involved in the G2/M cell cycle arrest by cisplatin.

Cisplatin treatment increases the expression levels of multiple autophagy-related genes. To investigate the mechanism involved in the autophagy-dependent cytotoxicity of cisplatin, we tested the expression levels of 29 genes closely associated with autophagy following treatment with cisplatin. ATG9B, ATG10, ATG16L1, RAB24 and RGS19 were not found to be expressed. Expression of ATG4A, ATG12 and LAMP1 was not changed. ATG4B, ATG9A and WIPI1 were downregulated, while AMBRA1, ATG3, ATG4C, ATG4D, ATG5, ATG7, ATG13, ATG14, ATG16L2, Beclin1, DRAM1, GABARAP, GABARAPL1, GABARAPL2, HDAC6, IRGM, MAP1LC3B and ULK1 were upregulated, and most of the upregulations were $>1.5$-fold (Fig. 5A).

\section{Discussion}

Autophagy is a homeostatic and evolutionarily conserved process that degrades cellular organelles and proteins, and maintains cellular biosynthesis during nutrient deprivation or metabolic stress (13). In the present study, we found that induction of autophagy was involved in the cytotoxicity of cisplatin. Mechanistically, cisplatin binds to and causes crosslinking of DNA, which repressed DNA replication and ultimately triggers apoptosis. Consistent with the repression on DNA replication, we found that treatment with cisplatin reduced accumulation of cells at the $\mathrm{S}$ phase. Notably, prolonged treatment increased the population of cells in the G2/M phase, suggesting that suppression of DNA replication is not the solo mechanism involved in the cytotoxicity of cisplatin. Considering the close link between autophagy and G2/M cell cycle arrest (11), we speculated that the autophagic pathway could be involved in the cisplatin-triggered $\mathrm{G} 2 / \mathrm{M}$ cell cycle arrest and cytotoxicity.

Autophagy is a complex process (Fig. 5B) that is divided into several distinct phases controlled by the autophagyrelated (ATG) family of proteins (14). Autophagy begins with the formation of double-membrane vesicles, known as autophagosomes, that engulf cytoplasmic constituents. The autophagosome then fuses with lysosomes, where the sequestered contents undergo degradation and recycling (13). The formation of the autophagosome could be divided into four stages: induction, nucleation (isolation of the membrane), elongation and completion of the double-membrane vesicle. In the present study, we assessed the mRNA expression of a range of genes closely associated with autophagy following treatment with cisplatin. Several genes participating in the formation of the autophagosome was clearly upregulated. These genes included AMBRA1, ATG3, ATG4C, ATG4D, ATG5, ATG7, ATG13, ATG14, ATG16L2, Beclin1, DRAM1, GABARAP, GABARAPL1, GABARAPL2, HDAC6, IRGM, MAP1LC3B and ULK1, suggesting that cisplatin induced autophagy by affecting multiple steps of autophagosome formation.
The induction stage of autophagy requires the ULK1 kinase complex, which consists of ULK1 (termed as ATG1 in yeast and ULK1 in mammals), ATG13 and FIP200 (15). ATG13 binds ULK1 and mediates the interaction of ULK1 with FIP200. The ULK1 kinase complex is a critical positive regulator of autophagosome formation. In the present study, both ULK1 and ATG13 were upregulated after treatment with cisplatin, suggesting the induction stage of autophagy was activated.

The vesicle nucleation stage depends on a complex containing Beclin1 (termed as Atg6 in yeast and Beclin1 in mammals), VPS34 and ATG14 (16). Stimulation of this complex generates phosphatidylinositol-3-phosphate (PI3P), which promotes autophagosomal membrane nucleation. Decreased expression of Beclin1 has been found in several types of cancers, including non-small cell lung (17) and breast cancer (18). Overexpression of Beclin1 represses cell proliferation, whereas knockdown of Beclin1 boosts cell growth and inhibits apoptosis (17). In our previous study, autophagy inducer artesunate repressed the growth of breast cancer cells through overexpression of Beclin1. In the present investigation, both Beclin1 and ATG14 were found to be overexpressed after cisplatin treatment. In addition, the activating molecule in Beclin1-regulated autophagy (AMBRA1), which was found to be a positive regulator of the Beclin1-dependent programme of autophagy (19), was overexpressed upon treatment with cisplatin, suggesting the participation of cisplatin in the nucleation stage of autophagy.

The elongation stage requires the participation of two ubiquitin-like protein conjugation systems, ATG12-ATG5 and LC3-phosphatidylethanolamine (PE). The ATG12-ATG5 conjugation system is a large multimeric complex, in which ATG12 is conjugated to ATG5 by ATG7 (an E1-like enzyme) and ATG10 (an E2-like enzyme), and then forms a complex with ATG16L. In the present study, ATG5 and ATG16L2 were found to be significantly upregulated and expression of ATG7 was also increased slightly, indicating promotion of the formation of the ATG12-ATG5 complex.

LC3 (as termed as ATG8) is a credible marker for autophagosomes. Mammalian LC3 homologs includes MAP1LC3A (LC3A), MAP1LC3B (LC3B), MAP1LC3C (LC3C), GABARAP, GABARAPL1 and GABARAPL2 (20). During the formation of autophagosomes, LC3 forms punctate structures within the cytoplasm that correspond to autophagic vesicles (9). In the LC3-PE conjugation system, LC3 is cleaved by the protease ATG4 to generate the LC3-I form. PE is conjugated to cleaved LC3 by an ATG7- and ATG3-dependent activation and transfer cascade to fulfill the conversion of LC3-I (a cytosolic truncated form) to LC3-II (autophagosomal membrane-associated, PE-conjugated form) (21). LC3-II binds with newly formed complete double-membrane autophagosomes and remains on mature autophagosomes until after fusion with lysosomes into the autophagolysosomes. Thereby LC3-II is commonly used to monitor autophagy. In the present study, overexpression of ATG4A, ATG4C, ATG4D, GABARAP, GABARAPL1, GABARAPL2 and MAPILC3B was observed. Moreover, damage-regulated modulator of autophagy (DRAM1), a lysosomal protein that induces autophagy through lysosomal acidification, fusion of lysosomes with autophagosomes and clearance of 
autophagosomes (22), was shown to be overexpressed as well. Mature autophagosomes then fuse their external membranes with those from lysosomes to degrade their cargo and recycle essential biomolecules. Finally, the ATG protein complexes from matured autophagosomes disintegrate (23).

The relationship between autophagy and tumorigenesis has been extensively explored $(24,25)$. However, whether autophagy is protumorigenic or antitumorigenic is still contradictory. In fact, autophagy is either cancer-promoting or cancer-suppressive, depending on the cell type, developmental stage of cancer and the stimulator $(24,25)$. On the basis of genetic studies, the tumor-suppressive functions of autophagy are most apparent during tumor initiation (26). The requirement for autophagy becomes more apparent in later stages, during which tumor cells cope with microenvironmental stress (27). Some environmental cues (starvation, high temperature, low oxygen and hormonal stimulation) or intracellular stresses (damaged organelles, accumulation of mutant proteins and microbial invasion) activate signaling pathways that induce autophagy-promoting cancer cell survival in these severe environments (28). Inhibition of protumorigenic autophagy by genetic or pharmacological strategies succeeds in killing tumor cells and triggering apoptosis in vivo (13). Furthermore, the combination of autophagy inhibitors and chemotherapeutics displays synergistic effects against cancer cells (13). On the contrary, some effective anticancer agents, such as rapamycin (29) and nilotinib (8), have been shown to repress cancer cell growth through the induction of autophagy. In our previous study, we confirmed that artesunate, a derivative of the active component of the Chinese medicinal herb Artemisia апnи $\mathrm{L}$., repressed breast cancer growth through autophagy induction and exhibited a synergistic anticancer effect with chemotherapy (5). These investigations suggest that both inhibition and induction of autophagy could be potential strategies in cancer treatment depending on whether the participating autophagy is protumorigenic or antitumorigenic.

In the present study, both protumorigenic and antitumorigenic autophagy were observed upon treatment with chemotherapeutics. Autophagy inhibition strengthened the cytotoxicity of docetaxel, yet impaired the cytotoxicity of cisplatin, suggesting the docetaxel-induced autophagy could be protumorigenic, while the cisplatin-induced autophagy could be antitumorigenic. In our previous study, we found that artesunate, an autophagy inducer, did not present a synergistic effect with docetaxel. This could be explained by the present results, as docetaxel itself could induce protumorigenic autophagy. Therefore, therapeutic targeting of the autophagic pathway should take many factors into consideration, as its chemotherapeutic allies may also affect autophagy induction and this effect could be either protumorigenic or antitumorigenic.

\section{Acknowledgements}

This study was supported by the National Natural Science Foundation of China (grant nos. 81472296, 81101867, 81272542, 81200369 and 81372443), the China International Medical Foundation (grant no. CIMF-F-H001-057), the Special Foundation of Clinical Medicine of Jiangsu Provincial Bureau of Science and Technology (grant no. BL2014039), the Scientific Research Project of Jiangsu Provincial Bureau of Traditional Chinese Medicine (grant no. L213236), the Medical Scientific Research Project of Jiangsu Provincial Bureau of Health (grant no. Z201206), the Special Foundation of Wu Jieping Medical Foundation for Clinical Scientific Research (grant nos. 320.6753.1225 and 320.6750.12242), the Science and Education for Health Foundation of Suzhou for Youth (grant nos. SWKQ1003 and SWKQ1011), the Science and Technology Project Foundation of Suzhou (grant nos. SYS201112, SYSD2012137 and SYS201335), the Science and Technology Foundation of Suzhou Xiangcheng (grant nos. SZXC2012-70 and XJ201451), and a Project Founded by the Priority Academic Program Development of Jiangsu Higher Education Institutions.

\section{References}

1. Sasco AJ, Kaaks R and Little RE: Breast cancer: Occurrence, risk factors and hormone metabolism. Expert Rev Anticancer Ther 3: 546-562, 2003.

2. Ferlay J, Shin HR, Bray F, Forman D, Mathers C and Parkin DM: Estimates of worldwide burden of cancer in 2008: GLOBOCAN 2008. Int J Cancer 127: 2893-2917, 2010.

3. Tan AA, Mu AK, Kiew LV and Chen Y: Comparative secretomic and $\mathrm{N}$-glycoproteomic profiling in human MCF-7 breast cancer and HMEpC normal epithelial cell lines using a gel-based strategy. Cancer Cell Int 14: 120, 2014.

4. Carlson RW and McCormick B: Update: NCCN breast cancer Clinical Practice Guidelines. J Natl Compr Canc Netw 3 (Suppl 1): S7-S11, 2005.

5. Chen K, Shou LM, Lin F, Duan WM, Wu MY, Xie X, Xie YF, $\mathrm{Li} \mathrm{W}$ and Tao M: Artesunate induces G2/M cell cycle arrest through autophagy induction in breast cancer cells. Anticancer Drugs 25: 652-662, 2014

6. Yu L, McPhee CK, Zheng L, Mardones GA, Rong Y, Peng J, Mi N, Zhao Y, Liu Z, Wan F, et al: Termination of autophagy and reformation of lysosomes regulated by mTOR. Nature 465 : 942-946, 2010.

7. Li W, Xie L, Chen Z, Zhu Y, Sun Y, Miao Y, Xu Z and Han X: Cantharidin, a potent and selective PP2A inhibitor, induces an oxidative stress-independent growth inhibition of pancreatic cancer cells through G2/M cell-cycle arrest and apoptosis. Cancer Sci 101: 1226-1233, 2010.

8. Yu HC, Lin CS, Tai WT, Liu CY, Shiau CW and Chen KF: Nilotinib induces autophagy in hepatocellular carcinoma through AMPK activation. J Biol Chem 288: 18249-18259, 2013.

9. Chen Y, Azad MB and Gibson SB: Methods for detecting autophagy and determining autophagy-induced cell death. Can J Physiol Pharmacol 88: 285-295, 2010.

10. Sheen JH, Zoncu R, Kim D and Sabatini DM: Defective regulation of autophagy upon leucine deprivation reveals a targetable liability of human melanoma cells in vitro and in vivo. Cancer Cell 19: 613-628, 2011.

11. Capparelli C, Chiavarina B, Whitaker-Menezes D, Pestell TG, Pestell RG, Hulit J, Andò S, Howell A, Martinez-Outschoorn UE, Sotgia F, et al: CDK inhibitors (p16/p19/p21) induce senescence and autophagy in cancer-associated fibroblasts, 'fueling' tumor growth via paracrine interactions, without an increase in neoangiogenesis. Cell Cycle 11: 3599-3610, 2012.

12. Plaimee P, Weerapreeyakul N, Barusrux S and Johns NP: Melatonin potentiates cisplatin-induced apoptosis and cell cycle arrest in human lung adenocarcinoma cells. Cell Prolif 48: 67-77, 2015.

13. Yang ZJ, Chee CE, Huang S and Sinicrope FA: The role of autophagy in cancer: Therapeutic implications. Mol Cancer Ther 10: 1533-1541, 2011.

14. Carew JS, Kelly KR and Nawrocki ST: Autophagy as a target for cancer therapy: New developments. Cancer Manag Res 4: 357-365, 2012.

15. Jung CH, Jun CB, Ro SH, Kim YM, Otto NM, Cao J, Kundu M and Kim DH: ULK-Atg13-FIP200 complexes mediate mTOR signaling to the autophagy machinery. Mol Biol Cell 20: 1992-2003, 2009.

16. FU LL, Cheng Y and Liu B: Beclin-1: Autophagic regulator and therapeutic target in cancer. Int J Biochem Cell Biol 45: 921-924, 2013. 
17. Wang W, Fan H, Zhou Y, Duan P, Zhao G and Wu G: Knockdown of autophagy-related gene BECLIN1 promotes cell growth and inhibits apoptosis in the A549 human lung cancer cell line. Mol Med Rep 7: 1501-1505, 2013.

18. Negri T, Tarantino E, Orsenigo M, Reid JF, Gariboldi M, Zambetti M, Pierotti MA and Pilotti S: Chromosome band 17q21 in breast cancer: Significant association between beclin 1 loss and HER2/NEU amplification. Genes Chromosomes Cancer 49: 901-909, 2010.

19. Pattingre S, Espert L, Biard-Piechaczyk M and Codogno P: Regulation of macroautophagy by mTOR and Beclin 1 complexes. Biochimie 90: 313-323, 2008.

20. Tanida I, Ueno $T$ and Kominami E: In vitro assays of lipidation of mammalian Atg8 homologs. Curr Protoc Cell Biol 64: 11.20:11.20.1-11.20.13, 2014.

21. Ravikumar B, Sarkar S, Davies JE, Futter M, Garcia-Arencibia M, Green-Thompson ZW, Jimenez-Sanchez M, Korolchuk VI, Lichtenberg M, Luo S, et al: Regulation of mammalian autophagy in physiology and pathophysiology. Physiol Rev 90: 1383-1435, 2010.

22. Crighton D, Wilkinson S, O'Prey J, Syed N, Smith P, Harrison PR, Gasco M, Garrone O, Crook T and Ryan KM: DRAM, a p53-induced modulator of autophagy, is critical for apoptosis. Cell 126: 121-134, 2006.
23. Yang Z and Klionsky DJ: Mammalian autophagy: Core molecular machinery and signaling regulation. Curr Opin Cell Biol 22: 124-131, 2010.

24. Degenhardt K, Mathew R, Beaudoin B, Bray K, Anderson D, Chen G, Mukherjee C, Shi Y, Gélinas C, Fan Y, et al: Autophagy promotes tumor cell survival and restricts necrosis, inflammation, and tumorigenesis. Cancer Cell 10: 51-64, 2006.

25. Qu X, Yu J, Bhagat G, Furuya N, Hibshoosh H, Troxel A, Rosen J, Eskelinen EL, Mizushima N, Ohsumi Y, et al: Promotion of tumorigenesis by heterozygous disruption of the beclin 1 autophagy gene. J Clin Invest 112: 1809-1820, 2003.

26. Debnath J: The multifaceted roles of autophagy in tumors-implications for breast cancer. J Mammary Gland Biol Neoplasia 16: 173-187, 2011.

27. Roy S and Debnath J: Autophagy and tumorigenesis. Semin Immunopathol 32: 383-396, 2010.

28. Bao XX, Xie BS, Li Q, Li XP, Wei LH and Wang JL: Nifedipine induced autophagy through Beclin1 and mTOR pathway in endometrial carcinoma cells. Chin Med J 125: 3120-3126, 2012.

29. Zhu BS, Xing CG, Lin F, Fan XQ, Zhao K and Qin ZH: Blocking NF-kappaB nuclear translocation leads to p53-related autophagy activation and cell apoptosis. World J Gastroenterol 17: 478-487, 2011. 